\title{
Neuropsychiatric symptoms in multiple sclerosis
}

Andrew Sommerlad MBBS, $B S c, M R C P s y c h$, Gary Price $M B C h B, M R C P s y c h, M S c$, PhD, Anand Trip BSC, MBChB, MRCP, PhD

\begin{abstract}
Multiple sclerosis is associated with a high prevalence of psychiatric comorbidity, including affective, psychotic and cognitive disorders. In this review, Dr Sommerlad and colleagues discuss the epidemiology, aetiology and management of neuropsychiatric symptoms in multiple sclerosis, using case vignettes to demonstrate the wide range of clinical presentations.
\end{abstract}

\section{Introduction}

Multiple sclerosis (MS) is the commonest cause of neurological disability in young and middle-aged adults $^{1}$. The characteristic pathological features are demyelinating plaques, astrocytic gliosis and immune-mediated inflammation anywhere within the central nervous system, with the periventricular region, brainstem, juxtacortical region, optic nerves and spinal cord being particularly common targets. The neurological manifestations are diverse although usually reflect the function of the affected anatomical site, and the course of the illness is variable and initially difficult to predict with accuracy.

In $80-90 \%$ of cases, the illness is relapsing-remitting in nature with two thirds of these developing a secondary progressive form of the condition a variable number of years after onset and a third having a relapsing-remitting course which does not result in long-term serious disability; the remaining $10-20 \%$ of patients have a primary progressive course with gradually worsening symptoms ${ }^{2}$. The prognosis can be poor in the long-term but progression is frequently slow; recent evidence suggests a median of 28 years before a stick is required for walking ${ }^{3}$.

The UK annual incidence of MS is $10 / 100,000$ with a prevalence of $200 / 100,000^{4}$. There is estimated to be over 100,000 people suffering from the condition in the UK. The median age of onset is 31 years and it is approximately twice as common in women ${ }^{5}$. There is multifactorial aetiology with genetic and environmental factors shown to be important. There is $31 \%$ monozygotic concordance compared to $5 \%$ in dizygotic twins ${ }^{6}$ while the environmental influence is demonstrated by marked variations in prevalence in genetically homogenous populations ${ }^{7}$. Prevalence increases with increasing distance from the equator and those who emigrate in childhood have prevalence rates consistent with their adopted country. ${ }^{8}$

MS commonly presents with weakness, tingling or numbness in the limbs; diplopia, visual loss or impaired visual acuity; vertigo; dysarthria and ataxia; fatigue or bladder and bowel disturbance ${ }^{5}$. The array of presenting symptoms in MS can lead to diagnostic uncertainty and neuropsychiatric conditions such as somatisation disorder are sometimes suspected. A study demonstrated that $16 \%$ of patients with MS had been referred to a psychiatrist between the onset of neurological symptoms and diagnosis of MS. In spite of neurological symptoms being present in the majority of cases, these remained largely overlooked and diagnoses of conversion disorder and hysterical personality disorder were applied ${ }^{9}$. While neurological features are the most recognised manifestations of the 
condition, psychopathology, including affective, psychotic and cognitive disorders, is also common in MS. The identification of these symptoms is often more difficult and diagnosis can be delayed. Psychiatric symptoms can occur at onset or later in the MS disease process and initial psychiatric symptoms tend to improve later than neurological symptoms and frequently never resolve ${ }^{10}$.

In this review article, we will describe a range of neuropsychiatric disorders which can develop in MS, illustrated by case vignettes from our clinical practice, and consider the most appropriate management.

\section{Mood disorders}

\section{Depression}

Case vignette 1 - Major depressive disorder

Andrea is a 45 year old woman with a 5 year history of relapsing-remitting MS with limb sensory deficit being the predominant feature. She presented with three month history of low mood, reduced motivation and feelings of hopelessness associated with suicidal thoughts. Cognitive assessment was normal and treatment with sertraline $100 \mathrm{mg}$ daily and cognitive behavioural therapy was initiated with good therapeutic effect at four month follow-up.

Depressive symptoms are common in patients with significant disability but distinction should be made between sufferers with these symptoms and those with the syndrome of major depression, which is characterised by pervasive mood change, diurnal mood variation, altered sleep and appetite and distorted beliefs including feelings of guilt and worthlessness and suicidal ideation. The lifetime prevalence of major depression in MS, replicated in a number of studies, is around $50 \%$ with a point prevalence of $14 \%{ }^{11}$, which is higher than matched populations with non-neurological disability and around three times higher than the general population.

Due to the potential overlap between somatic symptoms of depression and features of MS, clinicians should use a screening tool which gives weight to depressive thoughts to identify patients at risk of depression that warrant more detailed clinical interview. The Beck Depression Inventory ${ }^{12}$ and Hospital Anxiety and Depression Scale ${ }^{13}$ are suitable and have been validated for patients with MS.

The identification of depression in people with MS is important as it is linked with lower quality of life, more functional impairment, poor cognitive function and reduced adherence to medication regimes $^{14}$ as well as higher rates of suicide than the general population. Suicidal ideation is common in MS with a quarter of patients considering suicide at some point during their illness. Young males within five years of diagnosis are at highest risk of completed suicide while presence and severity of depression, social isolation and alcohol abuse are other notable risk factors ${ }^{13}$.

Biological and psychosocial factors are implicated in the aetiology of depression in MS. Evidence suggests that the individual's adjustment to adversity and their coping strategies is more closely related to physical deterioration than the severity of depression; loss of recreational opportunities and poor quality relationships are also important ${ }^{15}$. Studies have not demonstrated significant correlation between depression and plaque load ${ }^{16}$ but the location of lesions is significant. A higher lesion load in the anterior temporal lobe and inferior medial prefrontal cortex of the dominant 
hemisphere as well as dominant anterior temporal lobe atrophy are associated with depression ${ }^{17}$. Genetic factors have not been shown to be relevant, while some have proposed that dysfunction in the hypothalamic-pituitary-adrenal axis underlies the increased risk of depression in MS.

There has been some concern in the past that the use of disease-modifying drugs (DMDs), particularly interferon- $\beta$, resulted in increased risk of depression and suicide. However, recent evidence has suggested that this is not the case and the value of interferon in maintaining remission in MS means that it should remain a treatment option in all individuals, including those with a past history of depression, but used with caution in those with severe depression. It is appropriate to screen high-risk individuals for depression following the initiation of treatment with DMDs and, if depression develops, the agent need not be stopped automatically, but depression should be treated using standard options ${ }^{18}$.

Several treatment options seem to be effective in treating depression in MS but the strength of evidence for any particular treatment is poor. Although open label trials and case-reports suggest efficacy of many different antidepressants in the treatment of depression in MS, there have been only two previous blinded randomised-controlled trials (RCT), of desipramine ${ }^{19}$ and paroxetine ${ }^{20}$, which have shown only a non-statistically-significant trend towards improvement in the drug groups compared to placebo. Electroconvulsive therapy (ECT) shows efficacy in severe depression but can result in exacerbation of symptoms of $\mathrm{MS}^{21}$. Results from trials of individual or group psychological treatments have shown more promise and cognitive-behavioural therapy (CBT) can be helpful in mild-moderate depression and as effective as treatment with the antidepressant sertraline ${ }^{22}$. A wellconducted RCT of telephone-administered CBT compared to a control group of supportive therapy by telephone demonstrated a significant reduction in cases of depression and depressive symptoms after therapy but the difference between the groups were no longer significant at one year follow$u^{23}$.

Treatment of depression in MS remains difficult and is poorly guided by evidence but the Golman Consensus Group recommends individualised treatment of moderate-severe depression with a combination of an antidepressant and a form of psychotherapy, either supportive, CBT or interpersonal therapy ${ }^{14}$.

\section{Bipolar affective disorder}

Co-occurrence of bipolar affective disorder (bipolar disorder) and MS is uncommon but prevalence of bipolar disorder in people with MS is at least double the expected rate ${ }^{24}$ and a number of potential explanations have been proposed although none fully explain the association. Some can be attributed to the mania-inducing properties of steroids but a shared genetic diathesis has also been proposed ${ }^{25}$. White matter hyperintensities are often reported in patients with bipolar disorder but these are not always seen and their aetiology is unclear. However, a study of patients with psychosis in MS demonstrated a predominance of plaques bilaterally in the temporal horn areas - this was common to both affective and schizophrenia-like presentations ${ }^{26}$. 
Management of bipolar disorder in MS is not guided by robust evidence but lithium and sodium valproate have previously been used with success. The diuretic effect of lithium in patients prone to bladder pathology and the potential for additional neurological impairment from lithium toxicity, however, necessitates caution in this patient group. The administration of exogenous steroids remains a mainstay of treatment in MS but care should be taken in those with a personal or family history of depression. The proven efficacy of lithium as prophylaxis against steroid-induced mania ${ }^{27}$ suggests that careful monitoring of mental state on a reduced steroid dose, along with the addition of lithium, is likely to prove effective if a manic episode occurs during treatment.

\section{Disorders of affect}

\section{Pseudobulbar affect}

Pseudobulbar affect (PBA), synonymous with the condition of pathological laughing and crying, is characterised by uncontrollable laughing and/or crying without the associated subjective feelings of happiness or sadness and usually without any discernable stressor. PBA is socially and occupationally disabling and distressing for patients and their families. It is seen in around $10 \%$ of MS patients and is associated with long disease duration, a progressive course, cognitive impairment and greater physical disability ${ }^{28}$. PBA is also seen in other neurological conditions, including Parkinson's Disease, Motor Neurone Disease and stroke. Cognitive assessment usually demonstrates poor performance on tasks involving prefrontal function. Structural brain changes predict pseudobulbar affect ${ }^{29}$; bilateral medial inferior frontal and bilateral parietal hyperintense lesions and brainstem hypointense lesions are seen.

Use of the Centre for Neurologic Study Emotional Lability Scale, a seven-item self report tool, is recommended for screening ${ }^{12}$. While treatment with small doses of amitriptyline, fluoxetine or levodopa has previously shown some benefit, new guidelines from the American Academy of Neurology propose the use of a combination of dextromethorphan and quinidine, which were shown in two RCTs to reduce emotional lability and significantly improve quality of life ${ }^{12}$.

\section{Euphoria}

Case vignette 2 - Euphoria
Jane, a 22 year old woman with secondary progressive MS characterised by optic neuritis and limb weakness, presented to
neurology outpatient clinic with her mother who was concerned about her inappropriate public behaviour; she would
react with extreme excitement to unremarkable situations. She was inappropriate and incongruously optimistic during
assessment while neuropsychological assessment showed mild frontal and subcortical deficit. Symptomatic treatment with
sodium valproate yielded little benefit.

In possibly the earliest large study of neuropsychiatric problems in MS in 1926, Cottrell and Kinnier Wilson reported the presence of euphoria in 63 of 100 consecutive cases of disseminated sclerosis at Queen Square ${ }^{30}$, although recent studies have suggested prevalence in MS sufferers to be around $10 \%^{31}$. Euphoria should not be confused with hypomania or mania seen in bipolar affective disorder. It is a fixed mental state change (rather than fluctuating as seen in mania) distinguished by unconcern over physical disability and incongruous optimism and is associated with more severe MS, greater physical disability, cognitive dysfunction, lack of insight and a severe, often frontal, brain 
lesion load. It is seen in around $10 \%$ of MS sufferers and is likely to be related to impairment of the self-regulatory orbito-frontal network.

\section{Psychotic disorders}

Case vignette 3 - Psychotic episode

Simon is a 26 year old man who presented to the emergency department following two possible seizures and encephalopathy. He became emotionally labile and developed persecutory delusional ideas that he was the target of a conspiratorial plot. The addition of risperidone $2 \mathrm{mg}$ BD was effective in controlling these symptoms. MRI brain showed multiple white matter lesions in the corpus callosum and temporal horns which enhanced with gadolinium and CSF analysis revealed oligoclonal bands. The initial diagnosis was possible acute disseminated encephalomyelitis but treatment with interferon-beta was initiated after a year when he developed a sensory spinal cord syndrome characteristic of MS.

Although psychosis in people with MS is a rare occurrence, studies suggest that the prevalence rate surpasses that expected by chance ${ }^{32}$. The reason for this is unclear; it may be due to direct effect of demyelinating lesions as patients with MS and psychosis are more likely to have plaques affecting the temporal horns bilaterally ${ }^{26}$ although a viral precursor to both conditions has also been proposed.

Affective and schizophrenia-like presentations are seen equally when MS patients become psychotic but the predominant presentation is one of positive psychotic symptoms (usually persecutory delusions) with relative lack of negative symptoms (apathy, restricted affect and thought ${ }^{26}$ ). In addition, compared to schizophrenia, MS-related psychosis tends to present later (mean age around 36 years) and there is quicker resolution of symptoms, fewer psychotic relapses and a better longterm outcome with $60 \%$ of patients having only one psychotic episode over six year follow-up ${ }^{26}$.

The evidence to favour a particular antipsychotic is scarce but atypical agents are preferred due to lower risk of adverse neurological effects; olanzapine, quetiapine and risperidone. As irritability and agitation is common in people with MS and psychosis, hospital admission is sometimes required and benzodiazepines are appropriate if added sedation is required.

\section{Cognitive Disorders}

\section{Case vignette 4 - Cognitive impairment}

Derek, a 20 year old man with a five year history of relapsing-remitting MS, was admitted to neuropsychiatry ward due to a two month history of disorganised behaviour at home. During assessment, his behaviour was bizarre at times and he displayed fixed, concrete thinking and impaired attention. He scored 30/30 on MMSE but was slow to answer some aspects of this screen. Formal neuropsychological testing demonstrated significant impairment in executive functioning and information processing speed with reduced naming and facial recognition.

Cognitive deficit in MS was described by Charcot in 1877 who noted that MS causes 'marked enfeeblement of the memory, conceptions are formed slowly and intellectual and emotional faculties are blunted in their totality ${ }^{33}$. Slowed cognitive processing is present early in the disease and can be seen in people with clinically isolated syndromes - a single episode of demyelination in one area of the central nervous system, eg optic neuritis or brainstem or spinal cord syndrome who had asymptomatic lesions elsewhere in the brain on Magnetic Resonance Imaging (MRI) ${ }^{34}$. In people with established MS, around $40 \%$ have cognitive abnormalities ${ }^{35}$. Risk factors for impaired cognitive function in MS are age greater than 40 years, low premorbid intelligence or educational 
attainment and depression and cognitive impairment tends to develop alongside worsening physical disability, disease duration and the onset of a progressive disease course ${ }^{35}$. Patients with a relapsingremitting disease course generally perform better than those with progressive illness in whom the predominant deficit is executive function, rather than memory which is affected in relapsing remitting disease ${ }^{36}$.

Cognitive dysfunction adversely affects daily functioning, employment and quality of life and is frequently missed in routine assessment; when a neurologist suspects cognitive impairment, they are correct in over $90 \%$ of cases but when they do not suspect cognitive impairment, they are wrong in half of cases ${ }^{37}$. Thorough neuropsychological assessment is time-consuming and costly so approaches have been proposed to assess those in greatest need. The Mini-Mental State Examination is not useful in screening MS patients for cognitive impairment but screening using the Multiple Sclerosis Neuropsychological Screening Questionnaire ${ }^{37}$, a 15-item self- or informant-rated measure of cognitive and neuropsychiatric problems which has been validated for use in MS, may be a helpful strategy. More thorough assessment using a battery of neuropsychological tests which assess the cognitive domains most affected in MS patients (processing speed, learning and memory, executive function, visuospatial processing and word retrieval) is recommended for people in whom cognitive dysfunction is suspected; the Minimal Assessment of Cognitive Function in MS battery is a valid approach to neuropsychological assessment in MS patients ${ }^{38}$.

MS has been suspected of causing a subcortical pattern of cognitive deficit (ie forgetfulness, emotional or personality changes, slowed thought processes and impaired executive function) as it affects classic subcortical white matter tracts such as periventricular white matter tracts and the corpus callosum and this has been borne out by research; information processing speed, verbal memory, cognitive flexibility and executive function are all impaired ${ }^{39}$.

The presentation of cognitive deficit in MS is variable. Cognitive function tends not to vary with other markers of relapse and remission and the syndrome of a rapidly progressive dementia is rare but there is usually a progressive course with more cognitive domains affected, and correlation with markers of physical disability and social dysfunction ${ }^{40}$. Some patients can present with predominant severe cognitive impairment without significant impairment in other neurological domains and in these patients, brain atrophy is commonly seen on neuroimaging and DMDs are frequently ineffective ${ }^{41}$.

Cognitive impairment can be predicted on traditional MRI imaging by brain atrophy but not by the degree or location of demyelinating lesions ${ }^{42}$ although more advanced MRI techniques are showing more promise in this regard. The combination of structural and functional MRI imaging has demonstrated altered cerebral activation in MS patients performing as well as controls on tasks requiring attention while MS patients who performed worse on a planning ability task did not have abnormal brain activation, suggesting that compensatory mechanisms related to neuronal plasticity can mitigate the pathological changes but can become overwhelmed, leading to the manifestation of cognitive symptoms $\mathrm{s}^{35}$. There is also increasing evidence that grey matter pathology, reflected by atrophy, is implicated in cognitive dysfunction as MS progresses ${ }^{43}$. 
DMDs are the first-line of treatment for MS and secondary outcome data from some large RCTs have demonstrated that early treatment with interferon beta results in better processing speed and working memory ${ }^{44}$ but no RCTs have assessed this as a primary outcome so the methodology does not allow sound conclusions to be drawn. Initial enthusiasm for the use of acetyl-cholinesterase inhibitors or memantine, which are used in dementia, has dispersed as they have been shown to be ineffective ${ }^{45}$. Symptomatic treatment with the stimulants L-amphetamine and methylphenidate have been proposed by some authors but this is controversial ${ }^{46}$.

Of non-pharmacological treatments, cognitive rehabilitation is likely to be the most effective and has two components; restoration of function and compensation for loss of function. Relying on neuronal plasticity, restorative approaches aim to identify specific cognitive deficits and introduce remedial therapies to increase performance in a given area, such as graded practice for improving memory. Compensatory strategies aim to maximise the use of the retained cognitive abilities in order to improve social and occupational function, such as using notebooks or calendars to record important information or appointments and developing time management skills ${ }^{35}$.

\section{Conclusions}

An understanding of neuropsychiatric disorders in MS is important for all professionals involved in the care of people with MS. These disorders occur early in the disease, affect about half of patients at some point and have a significant impact on social/occupational function and quality of life. Furthermore, they negatively affect patients' ability to adhere to the recommended treatment regimens for other neurological symptoms in MS. Timely detection is essential as therapies can often be successful and improve quality of life, although the paucity of empirical treatment data demonstrates the need for targeted research on the management of these disorders.

\section{Declaration of interests}

None related to the subject of this article.

\section{Author roles}

Dr Sommerlad is a clinical training fellow in Old Age Psychiatry at University College London and Camden \& Islington NHS Foundation Trust. Dr Price is a Consultant Neuropsychiatrist \& Honorary Senior Lecturer and Dr Trip is a Consultant Neurologist \& Honorary Senior Lecturer at the National Hospital for Neurology and Neurosurgery, Queen Square, UCLH NHS Foundation Trust, London.

\section{Figures}

Table 1

\begin{tabular}{|l|l|}
\hline Useful Assessment Schedules \\
\hline Depression & Beck Depression Inventory, Hospital Anxiety and Depression Scale \\
\hline Pseudobulbar Affect & Centre for Neurologic Study Emotional Lability Scale \\
\hline Cognitive function Screening & Multiple Sclerosis Neuropsychological Screening Questionnaire \\
\hline Neuropsychological assessment & Minimal Assessment of Cognitive Function in MS \\
\hline
\end{tabular}

Table 2

\begin{tabular}{|l|l|}
\hline Treatment options in multiple sclerosis \\
\hline Depression & Sertraline, CBT \\
\hline Mania & Lithium, Sodium valproate \\
\hline Pseudobulbar palsy & Dextromethorphan and Quinidine \\
\hline
\end{tabular}




\begin{tabular}{|l|l|}
\hline Psychosis & Olanzapine, Quetiapine, Risperidone \\
\hline Cognitive impairment & Disease modifying drugs. Cognitive rehabilitation \\
\hline
\end{tabular}

Table 3

Key Points

Neuropsychiatric disorders are common in MS, especially depression and cognitive impairment which affect around 50\% of individuals at some point.

They can occur at any time in the disease process but are rarely the first presenting symptom.

Untreated neuropsychiatric symptoms result in worse social and occupational function and reduced quality of life.

Neuropsychiatric are under-recognised but appropriate screening and diagnostic tools are available.

There should be close liaison between psychiatry and neurology teams to achieve holistic management of these disorders. 


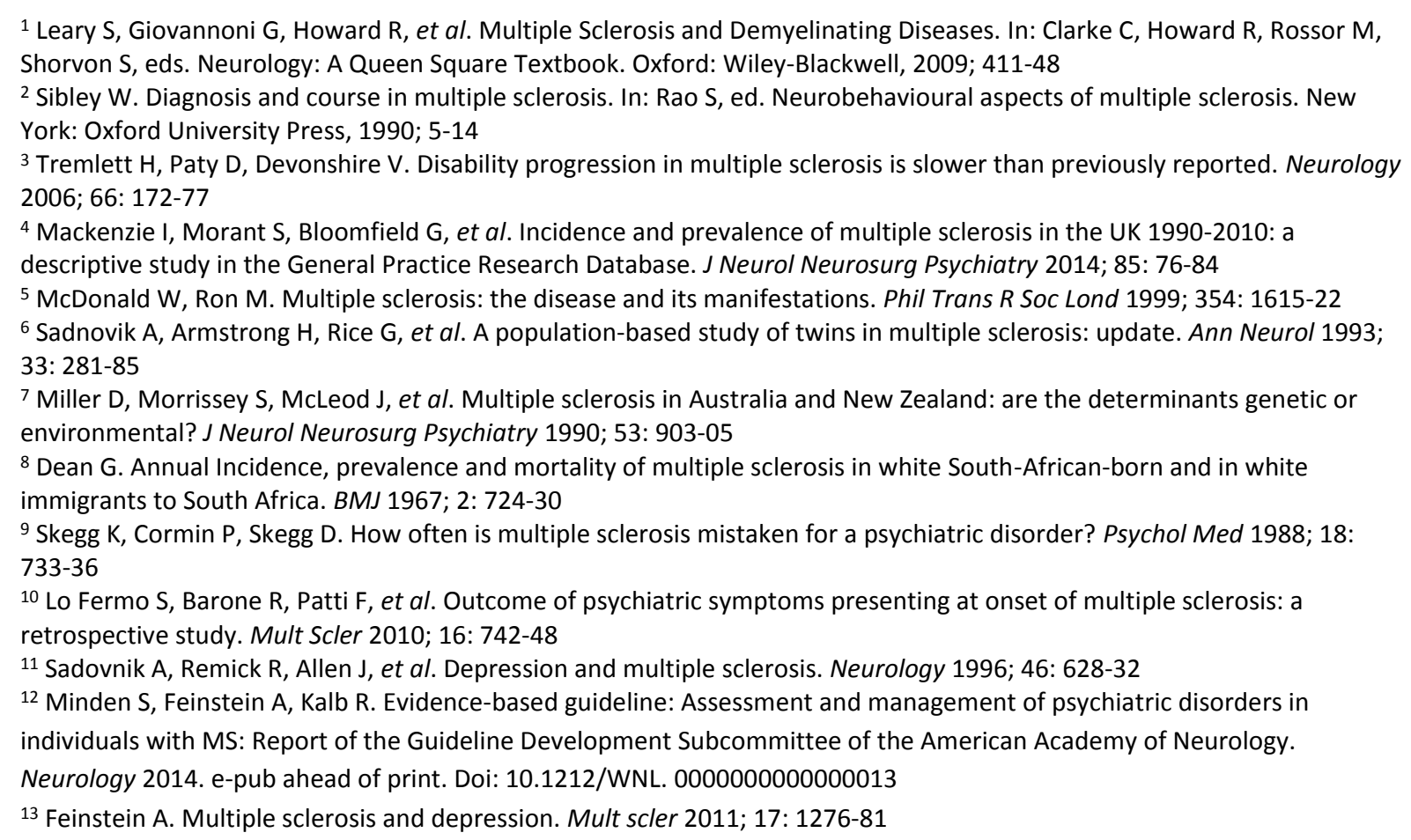

${ }^{14}$ Golman Consensus Group. The Goldman Consensus Group statement on depression in multiple sclerosis. Mult Scler 2005; 11: $328-37$

${ }^{15}$ Lynch S, Kroencke D, Denney D. The relationship between disability and depression in multiple sclerosis: the role of uncertainty, coping and hope. Mult Scler 2001; 7: 411-16

${ }^{16}$ Ron M, Logsdail S. Psychiatric morbidity in multiple sclerosis: a clinical and MRI study. Psychol Med 1989; 19: 887-95

17 Vattakatuchery J, Rickards H, Cavanna A. Pathogenic mechanisms of depression in multiple sclerosis. J Neuropsychiatry Clin Neurosci 2011; 23: 261-76

18 Goeb J-L, Even C, Nicolas G, et al. Psychiatric side effects of interferon- $\beta$ in multiple sclerosis. Eur Psychiatry 2006; 21: 186-93

${ }^{19}$ Schiffer R, Wineman N. Antidepressant pharmacotherapy of depression associated with multiple sclerosis. Am J Psychiatry 1990; 147: 1493-97

${ }^{20}$ Ehde D, Kraft G, Chwistiak L, et al. Efficacy of paroxetine in treating major depressive disorder in persons with multiple sclerosis. Gen Hosp Psychiatry 2008; 30: 40-48

${ }^{21}$ Krystal A, Coffey C. Neuropsychiatric considerations in the use of electroconvulsive therapy. J Neuropsychiatry Clin Neurosci 1997; 9: 283-92

22 Mohr D, Boudewyn A, Goodkin D, et al. Comparative outcomes for individual cognitive-behavioural therapy, supportiveexpressive group psychotherapy, and sertraline for the treatment of depression in multiple sclerosis. J Consult Clin Psychol 2001; 69: 942-49

${ }^{23}$ Cosio D, Jin L, Siddique J, et al. The effect of telephone-administered cognitive-behavioural therapy on quality of life among patients with multiple sclerosis. Ann Behav Med 2011; 41: 227-34

${ }^{24}$ Schiffer R, Wineman N, Weitcamp L, et al. Association between bipolar affective disorder and multiple sclerosis. Am J Psychiatr 1986; 143: 94-95

${ }^{25}$ Kosmidis M, Bozikas V, Giannouli V, et al. Familial comorbidity of bipolar disorder and multiple sclerosis: Genetic susceptibility, coexistence or causal relationship? Behav Neurol 2012; 25: 341-49

${ }^{26}$ Feinstein A, Du Bourlay G, Ron M. Psychotic illness in multiple sclerosis. A clinical and magnetic resonance imaging study. BJ Psych 1992; 161: 680-85

${ }^{27}$ Falk W, Mahnke M, Poskanzer D. Lithium prophylaxis of corticotropin-induced psychosis. J Amer Med Assoc 1979; 24:

1011-12

${ }^{28}$ Wortzel H, Oster T, Anderson C, et al. Pathological laughing and crying: epidemiology, pathophysiology and treatment. CNS Drugs 2008; 22: 531-45

${ }^{29}$ Ghaffar O, Chamelian L, Feinstein A. Neuroanatomy of pseudobulbar affect: a quantitative MRI study in multiple sclerosis. J Neurol 2008; 253: 406-12

${ }^{30}$ Cottrell S, Kinnier Wilson S. The affective symptomatology of disseminated sclerosis. J Neurol Psychopathol 1926; 7: 1-30

${ }^{31}$ Chiaravalloti N, Deluca J. Assessing the behavioral consequences of multiple sclerosis: an application of the Frontal

Systems Behavior Scale (FrSBe). Cogn Behav Neurol 2003; 16: 54-67 
32 Patten S, Svenson L, Metz L. Psychotic disorders in MS: population-based evidence of an association. Neurology 2005; 65: 1123-25

${ }^{33}$ Charcot J-M. Lectures on the diseases of the Nervous System delivered at La Salpetriere. London: New Sydenham Society, 1877 ; $194-95$

${ }^{34}$ Callanan M, Logsdail S, Ron M, et al. Cognitive impairment in patients with clinically isolated lesions of the type seen in multiple sclerosis: a psychometric and MRI study. Brain 1989; 112: 361-74

${ }_{35}$ Messinis L, Kosmidis M, Lyros E, et al. Assessment and rehabilitation of cognitive impairment in multiple sclerosis. Int Rev Psychiatr 2010; 22: 22-34

36 Zakzanis K. Distinct neurocognitive profiles in multiple sclerosis subtypes. Arch Clin Neuropsych 2000; 15: 115-36

${ }^{37}$ Benedict R. Integrating cognitive function screening and assessment into the routine care of multiple sclerosis patients. CNS Spectr 2005; 10: 384-91

${ }^{38}$ Benedict R, Cookfair D, Gavett R, et al. Validity of the Minimal Assessment of Cognitive Function in Multiple Sclerosis. J Int Neuropsych Soc 2006; 12: 549-58

${ }^{39}$ Calabrese P. Neuropsychology of multiple sclerosis: An overview. J Neurol 2006; 253: 10-15

${ }^{40}$ Feinstein A. The natural history of cognitive change in MS. In: Feinstein A, ed. The clinical neuropsychiatry of multiple sclerosis $2^{\text {nd }}$ edition. Cambridge: Cambridge University Press, 2007; 145-54

${ }^{41}$ Staff N, Lucchinetti C, Keegan B. Multiple sclerosis with predominant, severe cognitive impairment. Arch Neurol 2009; 66: $1139-43$

42 Benedict R, Weinstock-Guttman B, Fishman I, et al. Prediction of neuropsychological impairment in multiple sclerosis. Comparison of conventional magnetic resonance imaging measures of atrophy and lesion burden. Arch Neurol 2004; 61: 226-30

43 Penny S, Summers M, Swanton J, et al. Changing associations between cognitive impairment and imaging in multiple sclerosis as the disease progresses. J Neuropsychiatry Clin Neurosci 2013; 25: 134-40

${ }^{44}$ Kappos L, Freedman M, Polman C, et al. Effect of early versus delayed interferon beta 1-b treatment on disability after a first clinical event suggestive of multiple sclerosis: A three year follow-up analysis of the BENEFIT study. Lancet 2007; 370: 389-97

${ }^{45}$ Krupp LB, Christodoulou C, Melville P. Multicenter randomized clinical trial of donepezil for memory impairment in multiple sclerosis. Neurology 2011; 76: 1500-7

46 Patti F. Treatment of cognitive impairment in patients with multiple sclerosis. Expert Opin Investig Drugs 2012; 21: 1679- 